\title{
On a Question of Abraham Robinson's
}

\author{
Jochen Koenigsmann \\ Accepted for publication in Israel Journal of Mathematics
}

\begin{abstract}
In this note we give a negative answer to Abraham Robinson's question whether a finitely generated extension of an undecidable field is always undecidable. We construct 'natural' undecidable fields of transcendence degree 1 over $\mathbb{Q}$ all of whose proper finite extensions are decidable. We also construct undecidable algebraic extensions of $\mathbb{Q}$ that allow decidable finite extensions 1
\end{abstract}

A field $F$ is said to be decidable if $\operatorname{Th}(F)$, the first order theory of $F$ in the language $\mathcal{L}:=\{+, \times ; 0,1\}$ of rings, is decidable. Equivalently, $F$ is decidable if there is an effective axiomatization of $\operatorname{Th}(F)$, i.e., an algorithm producing a (typically infinite) list of $\mathcal{L}$-sentences true in $F$ from which every sentence in $\operatorname{Th}(F)$ can be deduced.

It is straightforward to see that if $F$ is decidable and $E$ is a finite extension of $F$ obtained by adjoining elements algebraic over the prime field that then $E$ is decidable as well.

40 years ago, in his retiring presidential address $[R]$ at the annual ASL meeting in Dallas, Abraham Robinson asked the following question in the opposite direction: Is a finitely generated extension of an undecidable field always undecidable? It turns out that the answer to this question is 'No' in a very strong sense:

Theorem 1. (A variation of Example 4.4 in [CDM] $]^{2}$ ) There are uncountably many pairwise non-elementarily-equivalent undecidable algebraic extensions

\footnotetext{
${ }^{1}$ The research on this note was undertaken while the author enjoyed the hospitality of the American Institute of Mathematics (AIM) which has been greatly appreciated. The author would also like to thank Carlos Videla for pointing him to Robinson's question.

${ }^{2} \mathrm{~A}$ week after presenting this Theorem at AIM we learned (via private communication) that a very similar result had already been proved in the unpublished manuscript [CDM],
} 
F of $\overline{\mathbb{Q}}(t)$ for which every proper finite extension $E / F$ is decidable (and all these $E$ are elementarily equivalent).

Here $\overline{\mathbb{Q}}$ denotes the algebraic closure of $\mathbb{Q}$ and $\overline{\mathbb{Q}}(t)$ the rational function field in one variable over $\overline{\mathbb{Q}}$.

In the light of this theorem it is natural to narrow down Robinson's question to fields that are algebraic over $\mathbb{Q}$, that is, to ask whether every finite extension of an undecidable algebraic extension $F$ of $\mathbb{Q}$ is always undecidable (a question also raised in the concluding Remark of [D]). This is known to be the case if $F$ is a number field, i.e., a finite extension of $\mathbb{Q}$ : all number fields are undecidable. In general, however, the answer is again in the negative:

Theorem 2. For every prime $p$ there are infinitely many pairwise nonisomorphic (and hence, here, non-elementarily-equivalent) decidable algebraic extensions $E$ of $\mathbb{Q}$ having uncountably many undecidable subfields $F$ of degree $[E: F]=p$.

While Robinson had asked his question about finitely generated field extensions $E / F$ we have, in both of the above theorems, even produced examples of finite, i.e., finitely generated algebraic field extensions $E / F$ such that $E$ was decidable and $F$ undecidable. As it happens, these examples were bound to be of this kind, as Robinson's question when asked about finitely generated non-algebraic field extensions is very likely to have a positive answer in the light of the following well supported:

Conjecture 3. If $F$ is an arbitrary field and $E / F$ a finitely generated nonalgebraic field extension then $E$ is undecidable.

Note that the conjecture does not assume $F$ to be undecidable.

This conjecture which has been raised by Malcev in $[\mathrm{M}]$ in the case that $E$ is a rational function field (so purely transcendental over $F$ ) has, for example, been proved in the following cases:

- if the characteristic of $F$ is $>0([\mathrm{ES}])$

- if $E$ is the function field of a curve over $F$, if $F$ is undecidable and either $F$ is large (in the technical sense that varieties over $F$ with one $F$-rational point have infinitely many) or, for some $n>1, F^{\times} /\left(F^{\times}\right)^{n}$ is finite: in this case, $F$ is definable in $E$ (Theorem 2 of $[\mathrm{K}]$ )

where the existence of an 'outlandish example' of an undecidable field $F$ of infinite transcendence degree over $\mathbb{Q}$ with all proper finite extensions being decidable and isomorphic has been established, though in a less constructive way. 
- if $E=F(t)$ and $F$ is formally real ([RR])

- if $E=F(t), F$ is infinite and existentially undecidable (for then $\operatorname{Th}_{\exists}(E)=$ $\left.\operatorname{Th}_{\exists}(F)\right)$

The most irritating unknown case is $\mathbb{C}(t)$ : it is not known whether or not $\mathbb{C}(t)$ is decidable.

As mentioned in the beginning, if $E / F$ is a finite extension that can be generated by elements algebraic over the prime field then decidability goes up (from $F$ to $E$ ). For arbitrary finite extensions $E / F$, however, in order to interpret $E$ in $F^{n}$ (where $n=[E: F]$ ) one needs parameters for the minimal polynomials of a basis for $E$ over $F$, yet it can happen that a field $F$ is decidable, but becomes undecicable if one allows new constants: For example if $F=\mathbb{R}$, any transcendental element $c \in \mathbb{R}$ which realizes an undecidable Dedekind cut, makes the theory with a constant for $c$ undecidable. We have no answer to the following twist of Robinson's question:

Question 4. Is a finite extension of a decidable field always decidable?

When Robinson presented his 'metamathematical problems' from twelve different areas of mathematics (the problem addressed in this note is only one of them) he emphasized that these problems seem of interest 'not only for their own sake but also because their solution might well require weapons whose introduction would close definite gaps in our armory'. The new weapons used in this note for solving the problem are, indeed, several deep results from field arithmetic, an area of mathematics that had rapidly developed only after Robinson's death in 1974.

For the convenience of the reader, in section 1, we shall recall these results which have by now become classics in their own right. Sections 2 and 3 are then devoted to proving Theorem 1 and 2 respectively.

Acknowledgement: The author would like to thank Moshe Jarden for suggesting very helpful improvements on an earlier draft. In particular, the proof of Proposition 9 is due to him.

\section{A few classics from field arithmetic}

Let us recall that a field $K$ is called Hilbertian if it satisfies Hilbert's Irreducibility Theorem, i.e., if for every irreducible polynomial $f(T, X) \in$ 
$K[T, X]$ there are infinitely many $t \in K$ such that $f(t, X) \in K[X]$ is irreducible (this property goes up to finite extensions of $K$ ).

$K$ is called PAC (pseudo algebraically closed) [resp. PRC (pseudo real closed)] if every non-empty variety $V$ defined over $K$ has a $K$-rational point [provided it has rational points in all real closures of $K$ ]. Both properties are inherited by any algebraic extension of $K$. If $K$ is not formally real then PRC and PAC are the same.

We denote the separable algebraic closure of $K$ by $\bar{K}$ and the absolute Galois group $\operatorname{Gal}(\bar{K} / K)$ by $G_{K}$.

Finally, we denote by $\hat{F}_{\omega}$ the free profinite group on countably infinitely many generators.

Fact 5. ([FV], also Example 24.8.5(b) in [FJ])

If $K$ is a countable Hilbertian PAC field then $G_{K} \cong \hat{F}_{\omega}$.

Conversely, it is also true that if $K$ is a countable PAC field with $G_{K} \cong \hat{F}_{\omega}$ then $K$ is Hilbertian (a result of Roquette, cf. Corollary 27.3.3 in [FJ])), a fact that we shall not use. What we will use, however, is the following celebrated result of Roquette's student Weissauer:

Fact 6. ([W], Satz 9.7, also Theorem 13.9.1(b) in [FJ])

Let $K$ be a Hilbertian field, let $F$ be a Galois extension of $K$ and let $F^{\prime}$ be a proper finite separable extension of $F$. Then $F^{\prime}$ is Hilbertian.

Fact 7. (A special case of a geometric local-global principle proved by MoretBailly in $[\mathrm{MB}]$, with a more elementary proof in [GPR])

Let $K$ be a countable Hilbertian field, let $\mathcal{P}$ be a non-empty finite set of orderings of $K$ and assume that either $K$ admits an ordering not in $\mathcal{P}$ or, for some prime $p, K$ can be embedded into $\mathbb{Q}_{p}$, the field of $p$-adic numbers. Let $K^{\mathcal{P}}$ be the intersection of all real closures of $K$ w.r.t. all orderings in $\mathcal{P}$. Then $K^{\mathcal{P}}$ is PRC and hence, by Fact $\left[, K^{\mathcal{P}}(\sqrt{-1})\right.$ is Hilbertian and PAC.

For the following characterization of $\hat{F}_{\omega}$ due to Iwasawa define a finite embedding problem for the profinite group $G$ to be a pair of (continuous) epimorphisms $(\phi: G \rightarrow A, \alpha: B \rightarrow A$ ) where $B$ (and hence $A$ ) is a finite group. A solution of the embedding problem is an epimorphism $\gamma: G \rightarrow B$ such that $\alpha \circ \gamma=\phi$ (sometimes these are called proper solutions while 'solutions' are not assumed to be onto). 
Fact 8. ([I], p. 567, also generalized in Theorem 24.8.1 in [FJ])

Let $G$ be a profinite group of at most countable rank. Then $G \cong \hat{F}_{\omega}$ if and only if every finite embedding problem for $G$ is solvable.

For a field $F$ of characteristic 0 we let $F^{a l g}:=F \cap \overline{\mathbb{Q}}$ denote its algebraic part and we let $\operatorname{Th}^{a l g}(F)$ be the subset of $\operatorname{Th}(F)$ that determines $F^{\text {alg }}$ (up to isomorphism) - it is axiomatized by saying which (irreducible, monic) polynomials in $\mathbb{Z}[X]$ have a zero in $F$ and which ones don't. Note that $\operatorname{Th}^{\text {alg }}(F)$, while determining $F^{\text {alg }}$ up to isomorphism, is still not an axiomatization of $\operatorname{Th}\left(F^{\text {alg }}\right): \operatorname{Th}^{\text {alg }}(F)$ is never complete.

Proposition 9. Let $F$ be a countable PAC field of characteristic 0 with $G_{F} \cong \hat{F}_{\omega}$. Then $F$ is decidable if and only if $\operatorname{Th}^{\text {alg }}(F)$ is decidable.

Proof: We extend the language $\mathcal{L}=\{+, \times ; 0,1\}$ of rings by predicates $R_{n}$, one for each $n \in \mathbb{N}$. Let $T_{R, 0}$ be the theory of fields of characteristic 0 with algebraic part $F^{\text {alg }}$ that satisfy all of the axioms

$$
R_{n}\left(X_{0}, \ldots, X_{n-1}\right) \longleftrightarrow(\exists Z)\left[Z^{n}+X_{n-1} Z^{n-1}+\cdots+X_{0}=0\right] .
$$

Let $L / K$ be an extension of fields of characteristic 0 with algebraic part $F^{a l g}$. Then $L / K$ is also an extension of models of $T_{R, 0}$ if and only if $K$ is algebraically closed in $L$. Since we are in characteristic 0 , the latter condition means that $L$ is a regular extension of $K$.

By a small variation of Theorem 27.2.3 of [FJ], $T_{R, 0}$ has a model companion $\widetilde{T}_{R, 0}$ whose models are $\omega$-free PAC-fields of characteristic 0 with algebraic part $F^{\text {alg }}$ (' $\omega$-free' means that any countable elementary substructure has absolute Galois group $\hat{F}_{\omega}$ ). By the preceding paragraph, $\widetilde{T}_{R, 0}$ has the amalgamation property. Hence $\widetilde{T}_{R, 0}$ is even a completion of $T_{R, 0}$. By Satz 3.22 of [Pr], it follows that $\widetilde{T}_{R, 0}$ admits elimination of quantifiers. Therefore, in order to decide whether a sentence in our extended language holds in $F$, we have to be able to decide which sentences of the form $R_{n}\left(a_{0}, \ldots, a_{n-1}\right)$ with $a_{0}, \ldots, a_{n-1} \in \mathbb{Z}$ hold in $F$, i.e., which polynomials $Z^{n}+a_{n-1} Z^{n-1}+\cdots+a_{0}$ have a root in $F^{\text {alg }}$. This is doable if and only if $\operatorname{Th}^{\text {alg }}(F)$ is decidable.

\section{Proof of Theorem 1}

Let $K$ be an algebraic extension of $\overline{\mathbb{Q}}(t)$ which is Hilbertian and PAC. Such extensions exist: for example, let $R:=\mathbb{R} \cap \overline{\mathbb{Q}}$ be the field of real algebraic 
numbers and let $\mathcal{P}$ be a non-empty finite set of orderings on $R(t)$. Then, by Fact 7 , the field $K:=R(t)^{\mathcal{P}}(\sqrt{-1})$ is Hilbertian, PAC and, by construction, an algebraic extension of $\overline{\mathbb{Q}}(t)$ (note that $R(t)$ allows infinitely many orderings) 3

Let $\Sigma$ be any set of primes and let $K(\Sigma)$ be the compositum of all $K(p)$ for $p \in \Sigma$ (inside a fixed algebraic closure of $K$ ), where $K(p)$ denotes the maximal pro- $p$ Galois extension of $K$.

Now construct a chain of algebraic extensions of $K$

$$
K_{0} \subseteq K_{1} \subseteq K_{2} \subseteq \ldots,
$$

where $K_{0}:=K$ and, for $n>0, K_{n}:=K_{n-1}(\Sigma)$. Then $F:=K_{\infty}(\Sigma):=$ $\bigcup_{n=0}^{\infty} K_{n}$ is a Galois extension of $K$ : note that $G_{K_{n}}$ is a characteristic subgroup of $G_{K_{n-1}}$, so all $G_{K_{n}}$ are normal (and, in fact, characteristic) subgroups of $G_{K}$. And, for any prime $p$,

$$
F=F(p) \Longleftrightarrow p \in \Sigma:
$$

' $\Leftarrow$ ' follows by construction. For ' $\Rightarrow$ ', assume $p \notin \Sigma$. As $K$ is Hilbertian, $K \neq K(p)$ and, by construction, $K(p) \cap F=K$. So $F \neq F K(p) \subseteq F(p)$.

In particular, if $\Sigma \neq \Sigma^{\prime}$ then $K_{\infty}(\Sigma)$ and $K_{\infty}\left(\Sigma^{\prime}\right)$ are not elementarily equivalent, so there are uncountably many pairwise non-elementarilyequivalent such $F$, of which only countably many can be decidable.

By Weissauer's Theorem (Fact 6), every proper finite extension $E / F$ is again Hilbertian (and as algebraic extension of the PAC field $K$ still PAC). Hence, by Fact 5, $G_{E} \cong \hat{F}_{\omega}$. Moreover, by construction, $E^{\text {alg }}=\overline{\mathbb{Q}}$, so $\operatorname{Th}^{\text {alg }}(E)$ is decidable (all $f \in \mathbb{Z}[X] \backslash \mathbb{Z}$ have zeros) and thus, by Proposition 9. so is $E$.

If one doesn't like the counting argument one may, alternatively, choose a set $\Sigma$ of primes which is not recursive in order to guarantee that $K_{\infty}(\Sigma)$ is not decidable.

\section{Proof of Theorem 2}

Let us first prove the following

\footnotetext{
${ }^{3}$ Alternatively, one could have used the fact that, (in the sense of the Haar measure on profinite groups) for almost all $\sigma \in G_{\overline{\mathbb{Q}}(t)}$, the maximal Galois extension $K$ of $\overline{\mathbb{Q}}(t)$ with $\sigma \in G_{K}$ is PAC and Hilbertian (Theorem 2.7 in [J], also Theorem 27.4.8 in [FJ]), but this is certainly less constructive.
} 
Lemma 10. Let $V$ be a vector space of infinite dimension over a field $k$, let $G$ be a (profinite) group acting on $V$ as group of $k$-linear transformations, asssume that all orbits of $V$ under this action of $G$ are finite and that, for every finite-dimensional $G$-invariant subspace $W$ of $V$, there is a non-zero $G$-invariant subspace $W^{\prime}$ of $V$ with $W \cap W^{\prime}=\{0\}$. Then, under the induced action of $G$ on the set $\widetilde{V}$ of all codimension 1 subspaces of $V, \widetilde{V}$ has uncountably many $G$-orbits.

Proof: Choose any $b_{1} \in V \backslash\{0\}$ and let $B_{1}$ be a maximal linearly independent subset of the (finite) $G$-orbit of $b_{1}$, so every element in the $G$-orbit of $b_{1}$ is in $\left\langle B_{1}\right\rangle$, the span of $B_{1}$. Now assume we have constructed finite sets $B_{1}, B_{2}, \ldots, B_{m}$ such that each $B_{i}$ spans the whole $G$-orbit of any of its members, $\bigcup_{i=1}^{m} B_{i}$ is linearly independent, and $W:=\left\langle B_{1} \cup \ldots \cup B_{m}\right\rangle=$ $\left\langle B_{1}\right\rangle \oplus \cdots \oplus\left\langle B_{m}\right\rangle$. Then, by assumption, there is a $G$-invariant non-zero subspace $W^{\prime}$ with $W \cap W^{\prime}=\{0\}$. So we can choose $b_{m+1} \in W^{\prime} \backslash\{0\}$ and let $B_{m+1}$ be a maximal linearly independent subset of the $G$-orbit of $b_{m+1}$. Then $B_{1} \cup \ldots \cup B_{m+1}$ is linearly independent and $\left\langle B_{1} \cup \ldots \cup B_{m+1}\right\rangle=B_{1} \oplus \cdots \oplus B_{m+1}$.

Now prolong the linearly independent set $\bigcup_{i=1}^{\infty} B_{i}$ to a basis $B$ of $V$ and define, for each proper subset $I$ of $\mathbb{N}$, a map $\chi_{I}: B \rightarrow k$ by setting

$$
\chi_{I}(b)= \begin{cases}0 & \text { if } b \in \bigcup_{i \in I} B_{i} \\ 1 & \text { if } b \in B \backslash \bigcup_{i \in I} B_{i}\end{cases}
$$

Let $T_{I}: V \rightarrow k$ be the $k$-linear map extending $\chi_{I}$. As $I$ was a proper subset of $\mathbb{N}$, the image of $T_{I}$ is $k$, and so $\operatorname{ker} T_{I}$ is a codimension 1 subspace of $V$, so $\operatorname{ker} T_{I} \in \widetilde{V}$. Moreover, for $I \neq I^{\prime}, \operatorname{ker} T_{I} \neq \operatorname{ker} T_{I^{\prime}}$ and, stronger still, $\operatorname{ker} T_{I}$ and $\operatorname{ker} T_{I^{\prime}}$ are in different $G$-orbits: otherwise there is some $\sigma \in G$ such that

$$
\operatorname{ker} T_{I^{\prime}}=\left\{\sigma(v) \mid v \in \operatorname{ker} T_{I}\right\}
$$

But then, say for $i \in I \backslash I^{\prime}$ and for any $b \in B_{i}, b \in \operatorname{ker} T_{I}$, and, since, by construction, $\sigma(b) \in\left\langle B_{i}\right\rangle$, also $\sigma(b) \in \operatorname{ker} T_{I}$, whereas $T_{I^{\prime}}(b)=1$ : contradiction.

As there are uncountably many proper subsets $I$ of $\mathbb{N}$, this shows that $\widetilde{V}$ has uncountably many distinct $G$-orbits.

To prove Theorem 2 , let $p$ be a given fixed prime. Let $K=\mathbb{Q}$ and let $\mathcal{P}$ be the singleton set containing the unique ordering on $\mathbb{Q}$. As $\mathbb{Q} \subseteq \mathbb{Q}_{l}$ for some (in fact, all) primes $l,(K, \mathcal{P})$ satisfies the hypotheses of Fact 7 and hence the field $L:=K^{\mathcal{P}}(\sqrt{-1})$ is Hilbertian and PAC (the field $K^{\mathcal{P}}=\mathbb{Q}^{\text {tot.r. }}$ 
is called the field of totally real numbers, it is the maximal Galois extension of $\mathbb{Q}$ inside $\mathbb{R}$ ). Now let

$$
E=L(\sqrt[p]{L}):=L(\{\sqrt[p]{a} \mid a \in L\})
$$

Then $E / L$ is a Galois extension ( $L$ contains a primitive $p$-th root $\zeta_{p}$ of unity, as $\zeta_{p}+\zeta_{p}^{-1} \in \mathbb{Q}^{\text {tot.r. }}$ and $\left.\sqrt{4-\left(\zeta_{p}+\zeta_{p}^{-1}\right)^{2}} \in \mathbb{Q}^{\text {tot.r. }}\right)$. As $L$ is Hilbertian,

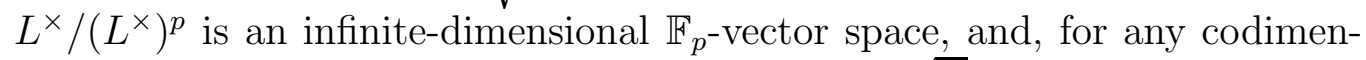
sion 1 subspace $U$ of $L^{\times} /\left(L^{\times}\right)^{p}$, the field $F_{U}:=L(\sqrt[p]{U})$ is a Galois subextension of $E / L$ with $[E: F]=p$. Hence, by Fact $[6, E$ is Hilbertian, and still PAC. By Fact 5, this implies that $G_{E} \cong \hat{F}_{\omega}$. Further note that $\operatorname{Th}^{\text {alg }}(E)$ is decidable: For each $n>0$ and each $\bar{a}=\left(a_{0}, \ldots, a_{n-1}\right) \in \mathbb{Z}^{n}$ let

$$
f_{\bar{a}}(X):=X^{n}+a_{n-1} X^{n-1}+\ldots+a_{1} X+a_{0} .
$$

Then the sets

- $I_{n}:=\left\{\bar{a} \in \mathbb{Z}^{n} \mid f_{\bar{a}}(X)\right.$ is irreducible $\}$,

- $T_{n}:=\left\{\bar{a} \in I_{n} \mid f_{\bar{a}}\right.$ splits in $\left.\mathbb{Q}^{\text {tot.r }}\right\}$ (that the splitting field of $f_{\bar{a}}$ is totally real is equivalent to $f_{\bar{a}}$ having $n$ distinct roots in $\mathbb{R}$ which, by (effective!) quantifier elimination for $\mathbb{R}$ in $\mathcal{L} \cup\{<\}$, is equivalent to some polynomial equations and inequalities in the coefficients $\bar{a}$ ), and

- $\mathcal{E}_{n}$ of those $\bar{a} \in I_{n}$ for which the splitting field of $f_{\bar{a}}$ is contained in an elementary abelian $p$-Galois extension of $M\left(\sqrt{-1}, \zeta_{p}\right)$ for some totally real Galois extension $M / \mathbb{Q}$ with $[M: \mathbb{Q}] \leq n$ !

are all decidable (= recursive) and hence, so is $\operatorname{Th}^{\text {alg }}(E)$, being axiomatized by

$$
\begin{aligned}
& \left\{\neg \exists x f_{\bar{a}}(x)=0 \vee \exists x_{1}, \ldots, x_{n} f_{\bar{a}}(X)=\prod_{i=1}^{n}\left(X-x_{i}\right) \mid \bar{a} \in I_{n}, n \in \mathbb{N}\right\} \\
\cup & \left\{\neg \exists x f_{\bar{a}}(x)=0 \mid \bar{a} \in I_{n} \backslash \mathcal{E}_{n}, n \in \mathbb{N}\right\} \\
\cup & \left\{\exists x_{1}, \ldots, x_{n} f_{\bar{a}}(X)=\prod_{i=1}^{n}\left(X-x_{i}\right) \mid \bar{a} \in \mathcal{E}_{n}, n \in \mathbb{N}\right\}
\end{aligned}
$$

Hence, by Proposition 9, $E$ is decidable.

To see that there are uncountably many $U$ as above for which the corresponding $F_{U}$ are pairwise non-elementarily equivalent, let $G:=\operatorname{Gal}(L / \mathbb{Q})$

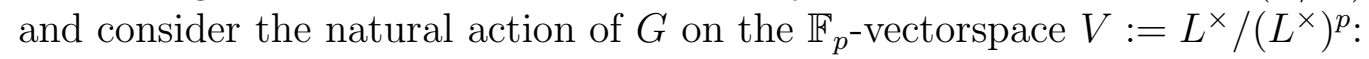

$$
\begin{aligned}
& G \times L^{\times} /\left(L^{\times}\right)^{p} \longrightarrow L^{\times} /\left(L^{\times}\right)^{p} \\
& \left(\sigma, a\left(L^{\times}\right)^{p}\right) \longmapsto(\sigma a)\left(L^{\times}\right)^{p}
\end{aligned}
$$


As each $a \in L^{\times}$lies in a finite Galois subextension $L_{a} / \mathbb{Q}$ of $L / \mathbb{Q}$, each $G$-orbit of $L^{\times} /\left(L^{\times}\right)^{p}$ is finite: the orbit of $a\left(L^{\times}\right)^{p}$ is $\left\{\tau(a) \cdot\left(L^{\times}\right)^{p} \mid \tau \in \operatorname{Gal}\left(L_{a} / \mathbb{Q}\right)\right\}$.

Moreover, for any finite(-dimensional) $G$-invariant subspace $W$ of $V$, there is a non-zero $G$-invariant subspace $W^{\prime}$ of $V$ with $W \cap W^{\prime}=\{0\}$ : If $p>2$ then the canonical $\mathbb{F}_{p}$-vector space homomorphisms

$$
\mathbb{Q}^{\times} /\left(\mathbb{Q}^{\times}\right)^{p} \longrightarrow \mathbb{Q}^{\text {tot.r. } \times} /\left(\mathbb{Q}^{\text {tot.r. } \times}\right)^{p} \longrightarrow L^{\times} /\left(L^{\times}\right)^{p}
$$

are all injective, so, for any $a \in \mathbb{Q}^{\times} \backslash\left(\mathbb{Q}^{\times}\right)^{p}$ with $a\left(L^{\times}\right)^{p} \notin W, W^{\prime}:=\left\langle a\left(L^{\times}\right)^{p}\right\rangle$ is a 1-dimensional $G$-invariant subspace of $V$ with $W \cap W^{\prime}=\{0\}$. If $p=2$, then for any prime $q, \sqrt{q}$ spans a 1-dimensional $G$-invariant subspace of $V: \sqrt{q}\left(L^{\times}\right)^{2}=(-\sqrt{q})\left(L^{\times}\right)^{2}$, so, again, we find $W^{\prime}$ as required: note that $\sqrt{q} \notin \pm\left(\mathbb{Q}^{\text {tot.r. } \times}\right)^{2}$ and that the kernel of $\mathbb{Q}^{\text {tot.r. } \times} /\left(\mathbb{Q}^{\text {tot.r. } \times}\right)^{2} \longrightarrow L^{\times} /\left(L^{\times}\right)^{2}$ is $\pm\left(\mathbb{Q}^{\text {tot.r. } \times}\right)^{2} /\left(\mathbb{Q}^{\text {tot.r. } \times}\right)^{2}$.

Now, by Lemma 10, there are uncountably many codimension 1 subspaces $U$ of $V$ all lying in distinct $G$-orbits. But for any two codimension 1 subspaces $U, U^{\prime}$ of $V$ one has

$$
\exists \sigma \in G U^{\prime}=\sigma(U) \Longleftrightarrow F_{U^{\prime}} \cong F_{U} \Longleftrightarrow F_{U^{\prime}} \equiv F_{U},
$$

so that there are uncountably many pairwise non-elementarily equivalent such $F_{U}$, as claimed.

This gives just one $E$ of the kind Theorem 2 wants to have it. To get infinitely many, let $l$ be any prime $\neq 2, p$ and let $E_{l}:=E(\sqrt[l]{2})$. Then $E_{l}$ (being parameter-free interpretable in $E^{l}$ ) is still decidable and there are uncountably many undecidable $F_{U, l}:=F_{U}(\sqrt[l]{2})$ with $U$ as above and $\left[E_{l}\right.$ : $\left.F_{U, l}\right]=p$. Finally, observe that $\sqrt[l]{2} \notin \mathbb{Q}^{\text {tot.r. }}$ (as $\left.l>2\right)$, so $\sqrt[l]{2} \notin E($ as $l \neq p)$ and, for $l \neq l^{\prime}, E_{l} \not \equiv E_{l^{\prime}}$.

\section{References}

[CDM] Gregory Cherlin, Lou van den Dries, Angus Macintyre, The elementary theory of regularly closed fields, manuscript, 1980.

[D] Lou van den Dries, New decidable fields of algebraic numbers, Proc. AMS 77(2) (1979), 251-256.

[ES] Kirsten Eisenträger, Alexandra Shlapentokh, Undecidability in function fields of positive characteristic, Int. Math. Res. Not. 2009 (2009), 4051-4086. 
[FJ] Michael Fried, Moshe Jarden, Field Arithmetic, Springer 1986, 3rd edition (revised by Moshe Jarden) 2008.

[FV] Michael Fried, Helmuth Völklein, The embedding problem over a Hilbertian PAC-field, Ann. Math. 135 (1992), 469-481.

[GPR] Barry Green, Florian Pop, Peter Roquette, On Rumely's local-global principle, Jber. Dt. Math.-Verein. 97 (1995), 43-74.

[J] Moshe Jarden, Large normal extensions of Hilbertian fields, Math. Zeitschr. 224 (1997), 555-556.

[K] Jochen Koenigsmann, Defining transcendentals in function fields, J. Symb. Logic 67(3) (2002), 947-956.

[M] Anatoli Ivanovic Malcev, On the undecidability of the elementary theory of certain fields (Russian), Sibrisk. Math. Zurnal 1(1) (1960), 71-77.

[MB] Laurent Moret-Bailly, Groupes de Picard et problèmes de Skolem II, Ann. Sci. École Norm. Sup. (4) 22 (1989), 181-194.

[Pr] Alexander Prestel, Einführung in die mathematische Logik und Modelltheorie, Vieweg 1986.

[R] Abraham Robinson, Metamathematical Problems, J. Symb. Logic 38(3) (1973), 500-516.

[RR] Raphael Robinson, The undecidability of pure transcendental extensions of real fields, Zeitschr. Math. Logik und Grundlagen Math. 10 (1964), 275-282.

[W] Rainer Weissauer, Der Hilbertsche Irreduzibilitätssatz, J. reine angew. Math. 334 (1982), 203-220.

Mathematical Institute, Radcliffe Observatory Quarter, Woodstock Road, Oxford OX2 6GG, UK

koenigsmann@maths.ox.ac.uk 